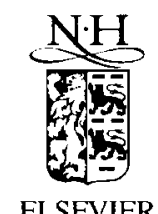

EJ SEVIER

\title{
Testing periodically integrated autoregressive models
}

\author{
Philip Hans Franses ${ }^{\mathbf{a}}$, Michael McAleer ${ }^{\mathbf{b}, *}$ \\ ${ }^{a}$ Econometric Institute, Erasmus University, Rotterdam, The Netherlands \\ ${ }^{\mathrm{b}}$ Department of Economics, University of Western Australia, Nedlands, WA 6907, Australia
}

\begin{abstract}
Periodically integrated time series require a periodic differencing filter to remove the stochastic trend. A non-periodic integrated time series needs the first-difference filter for similar reasons. When the changing seasonal fluctuations for the nonperiodic integrated series can be described by seasonal dummy variables for which the corresponding parameters are not constant within the sample, such a series may not be easily distinguished from a periodically integrated time series. In this paper, testing procedures developed by Franses and McAleer [4] are used to distinguish between these two alternative stochastic and non-stochastic seasonal processes when there is a single known structural break in the seasonal dummy parameters. Two empirical examples, namely, the logarithms of quarterly real GNP series for Austria and Germany, are used to illustrate the approach.
\end{abstract}

\section{Introduction}

One of the major debates in empirical macroeconomics focuses on the characteristic of seasonal fluctuations. An important question is whether constant seasonal patterns, which can be described using seasonal dummy variables, are adequate to describe seasonality, or whether changing seasonal patterns, which may be described using seasonal unit root processes, are preferable. A flexible class of time series models which can address this question is the periodically integrated (PI) time series model, a detailed exposition of which is given in Boswijk and Franses [1]. In particular, a PI time series of order 1 is defined by a seasonally varying differencing filter, which is required to remove the stochastic trend from the time series. The variation in the seasonal coefficients in the periodic differencing filter allows for multiplicative seasonality, and for exogenous shocks to have different impacts in different seasons.

Another empirical aspect of macroeconomic time series is that there can be structural breaks during the sample period. Franses and McAleer [4] argue that, if a PI process is found to yield an adequate description of a time series, it should be compared with a non-periodically integrated (or, simply, an integrated (I)) process with structural shifts in one or more of the seasonal means.

* Corresponding author 
In this paper, testing procedures developed by Franses and McAleer [4] are used to distinguish between an I process with non-constant parameters and a PI process. It is assumed that there is a single known one-time parameter change for the seasonal dummy parameters. Two empirical examples, namely, the logarithms of quarterly real GNP series for Austria and Germany, are used to illustrate the approach.

The outline of the paper is as follows. In Section 2, the two types of stochastic and non-stochastic seasonal models are highlighted. Alternative testing procedures are discussed in Section 3 , and are illustrated for two quarterly real GNP series in Section 4.

\section{Two seasonal models}

\subsection{A stochastic specification}

Consider a time series $y_{t}$ which is observed at quarterly intervals over $N$ years, where $t$ is the quarterly index running from $1 \ldots n$, and $n=4 N$. A flexible class of time series models that allows for seasonal unit roots, non-seasonal unit roots and periodic integration is the periodic autoregressive process (PAR). A PAR process of order $p, \operatorname{PAR}(p)$, can be represented by

$$
y_{t}=\mu_{s}+\phi_{1 s} y_{t-1}+\ldots+\phi_{p s} y_{t-p}+\varepsilon_{t}
$$

where the index $s$ denotes that the parameter values can vary over the four seasons, $s=1 \ldots 4$. The $\varepsilon_{t}$ are assumed to follow a standard white noise process. Estimation of the parameters in Eq. (1) is through using ordinary least squares on

$$
y_{t}=\sum_{s=1}^{4} D_{s t} \mu_{s}+\sum_{s=1}^{4} D_{s t}\left(\phi_{1 s} y_{t-1}+\ldots+\phi_{p s} y_{t-p}\right)+\varepsilon_{t}
$$

where the $D_{s t}$ are seasonal dummy variables which equal one in season $s$ and zero in all other seasons, and the number of parameters is $4(p+1)$.

In the case of a single unit root (i.e. a PI autoregressive time series of order $p$, or PIAR ( $p$ )), Eq. (1) can be written as

$$
\left(1-\alpha_{s} B\right) y_{t}=\mu_{s}+\beta_{1 s}\left(1-\alpha_{s-1} B\right) y_{t-1}+\ldots+\beta_{p-1, s}\left(1-\alpha_{s-p+1} B\right) y_{t-p+1}+\varepsilon_{t}
$$

subject to the non-linear restriction

$$
\alpha_{1} \alpha_{2} \alpha_{3} \alpha_{4}=1
$$

where $B$ is the familiar backward shift operator, the parameters $\alpha_{\mathrm{s}}$ and $\beta_{j s}$ are functions of the parameters $\phi_{p s}$ in Eq. (1), and the $1-\alpha_{s} B$ filter is called the periodic differencing filter. The model in Eq. (2) subject to Eq. (3) can be estimated using non-linear least squares. Since $\left(1-\hat{\alpha}_{s} B\right) y_{t}$ is a periodically stationary time series, where $\hat{\alpha}_{s}$ is the non-linear least squares estimator of $\alpha_{s}$, the $t$-ratios for the estimated $\beta_{j s}$ parameters in Eq. (2) have the asymptotic standard normal distribution.

It is clear from Eqs. (2) and (3) that, given Eq. (3), the ordinary integrated time series process that requires the $1-B$ filter to remove the stochastic trend is nested within Eq. (2). In that case, all the $\alpha_{s}$ are equal to 1, and hence Eq. (3) holds automatically. 


\subsection{A non-stochastic specification}

Franses and McAleer [4] argue that a variable alternative model arises where the seasonal patterns might be caused by deterministic shifts rather than by stochastic shifts. They contrast the PIAR model in Eqs. (2) and (3) with a model that considers the first-order differenced time series, $(1-B) y_{t}$, when it can be described using an (periodic) autoregression and allows for a known one-time structural break at given time $\tau$, namely,

$$
(1-B) y_{t}=\delta_{s}^{*}+\mathbf{I}_{(t \geq \tau)} \delta_{s}^{*}+\beta_{1 s}(1-B) y_{t-1}+\ldots+\beta_{p-1, s}(1-B) y_{t-p+1}+\varepsilon_{t}
$$

In Eq. (4) the variable $\mathrm{I}_{(t \geq \tau)}$ is an indicator function which is equal to 1 when $t \geq \tau$, and the number of parameters is $8+4(p-1)$. For convenience, it is assumed that the model remains periodic so that the $\beta$ parameters vary seasonally. The $\delta_{s}^{*}$ parameters have values that may be different from zero, which implies that the growth rates can change at time $\tau$. In the next section, testing procedures developed by Franses and McAleer [4] are discussed for choosing between Eqs. (2)-(4), when they are both nested within a more general model and also when they are non-nested.

\section{Testing procedures}

The two time series models in Eqs. (2)-(4), are such that Eq. (2) sets to zero the coefficient of the structural break dummy variable $\mathbf{I}_{(t \geq \tau)}$, whereas Eq. (4) imposes a unit root restriction rather than a periodic unit root restriction (and implicitly imposes the non-linear restriction given in Eq. (3)). Several procedures are available for testing each of these two models, depending on whether they are interpreted as non-nested models or as specializations of an interpretable more general model.

\subsection{Nested procedures}

When the two models Eqs. (2)-(4), may be regarded as specializations of a more general explanation, Franses and McAleer [4] suggest nesting both in the more general model, with $3+8+4(p-1)$ parameters, given by

$$
\left(1-\alpha_{s} B\right) y_{t}=\beta_{1 s}\left(1-\alpha_{s-1} B\right) y_{t-1}+\ldots+\beta_{p-1, s}\left(1-\alpha_{s-p+1} B\right) y_{t-p+1}+\kappa_{s}+\mathrm{I}_{(t \geq \tau)} \kappa_{s}^{*}+\varepsilon_{t}
$$

which is to be estimated subject to Eq. (3). It is assumed, in the first instance, that $\tau$ is known. The validity of Eq. (5) rests in its ability to accommodate both periodic integration and a structural break. It should be noted that the structural break is accommodated within the periodically integrated model, so that the non-linear restriction is imposed in estimating Eq. (5). The general model Eq. (5), in which all variables are (periodically) stationary, may be estimated using non-linear least squares.

The PI model in Eqs. (2) and (3) implies that there is no structural break, so that the $\kappa_{s}^{*}$ parameters in Eq. (5) are equal to zero, in which case the $\kappa_{s}$ are equal to the $\mu_{s}$ in Eq. (2). Since the $\kappa_{s}^{*}$ are coefficients of stationary variables (i.e. the corresponding variables have a value of one for $t \geq \tau$ ), the PI model can be compared with Eq. (5) using the standard $F$ test. This test will be denoted $F_{\mathrm{PI}}$ $(4, n-k)$, where $k$ is the number of parameters in the general model and PI in Eqs. (2) and (3) is the null hypothesis.

The integrated time series model Eq. (4) implies that each of the $\alpha_{s}$ in Eq. (5) is equal to one, in which case the non-linear restriction is satisfied automatically. When each $\alpha_{s}$ is equal to one, the $\kappa_{s}$ and 
$\kappa_{s}^{*}$ in Eq. (5) equal $\delta_{s}$ and $\delta_{s}^{*}$, respectively. Model Eq. (4) can be tested against Eq. (5) using the standard $F$ test, denoted as $F_{\mathrm{I}}(3, n-k)$, where $k=4 p+7$ in Eq. (5) and $\mathrm{I}$ is the null hypothesis. The three restrictions to be tested arise from the fact that Eq. (5) is estimated under the non-linear restriction in Eq. (3), in which case there are only three free $\alpha_{s}$ parameters.

The interpretation to be given to these test statistics is straightforward. A significantly large value of $F_{\mathrm{PI}}\left(F_{\mathrm{I}}\right)$ leads to the rejection of the PI (I) model in Eqs. (2)-(4), in favour of the general model with both periodic integration and a structural break. A significant test statistic for the null hypothesis of an integrated model with no structural break also leads to the general model Eq. (5). Insignificant test statistics in each of these three cases leads to non-rejection of the respective null hypotheses. It should be emphasized that, in the nested case, the general model given in Eq. (5) may not only be statistically valid, but may also be interpreted as a useful model in itself.

\subsection{Non-nested procedures}

When the standard $F$ test in a model such as Eq. (5) cannot be entertained, non-nested testing procedures may be used. Franses and McAleer[4] develop tests of Eqs. (2)-(4), against each other, based on the $J$-test procedure of Davidson and MacKinnon [3]. Using weights $(1-\lambda)$ and $\lambda$ for the models given in Eq. (2) and Eq. (4), respectively, yields

$$
\begin{aligned}
& (1-\lambda)\left(1-\alpha_{s} B\right) y_{t}+\lambda(1-B) y_{t}=(1-\lambda)\left[\mu_{s}+\beta_{1 s}\left(1-\alpha_{s-1} B\right) y_{t-1}\right. \\
& \left.\quad+\ldots+\beta_{p-1, s}\left(1-\alpha_{s-p+1} B\right) y_{t-p+1}\right]+\lambda\left[\delta_{s}+\mathbf{I}_{(t \geq \tau)} \delta_{s}^{*}+\beta_{1 s}(1-B) y_{t-1}\right. \\
& \left.\quad+\ldots+\beta_{p-1, s}(1-B) y_{t-p+1}\right]+\varepsilon_{t}
\end{aligned}
$$

which is estimated subject to Eq. (3). In Eq. (6), $\lambda$ is not identifiable without imposing restrictions. Since there are several methods for identifying $\lambda$ (see Ref. [6] for further details), the method adopted here is to use sample information. In order to test $H_{0}: \lambda=0$ in Eq. (6), estimates of the parameters from Eq. (4) are used to replace the unknown parameters as follows:

$$
\begin{aligned}
& (1-\lambda)\left(1-\alpha_{s} B\right) y_{t}+\lambda(1-B) y_{t}=(1-\lambda)\left[\mu_{s}+\beta_{1 s}\left(1-\alpha_{s-1} B\right) y_{t-1}\right. \\
& \left.\quad+\ldots+\beta_{p-1, s}\left(1-\alpha_{s-p+1} B\right) y_{t-p+1}\right]+\lambda\left[\hat{\delta}_{s}+\mathbf{I}_{(t \geq \tau)} \hat{\delta}_{s}^{*}+\hat{\beta}_{1 s}(1-B) y_{t-1}\right. \\
& \left.\quad+\ldots+\hat{\beta}_{p-1, s}(1-B) y_{t-p+1}\right]+\varepsilon_{t}
\end{aligned}
$$

which is to be estimated subject to Eq. (3). Since all the variables in Eq. (7) are (periodically) stationary, non-linear least squares applied to (7)-(3) yields a $t$-ratio for the estimate of $\lambda$ which is asymptotically distributed as standard normal. Let the $t$-ratio for the test of PI in Eqs. (2) and (3) against I in Eq. (4) be denoted as $t_{\mathrm{PI}}$.

A test of $H_{1}: \lambda=1$ in Eq. (6), namely, testing Eq. (4) against Eqs. (2) and (3), may be obtained by replacing the unknown parameters of Eq. (2) with estimates from Eqs. (2) and (3) as follows:

$$
\begin{aligned}
(1- & \lambda)\left(1-\hat{\alpha}_{s} B\right) y_{t}+\lambda(1-B) y_{t} \\
= & (1-\lambda)\left[\hat{\mu}_{s}+\hat{\beta}_{1 s}\left(1-\hat{\alpha}_{s-1} B\right) y_{t-1}+\ldots+\hat{\beta}_{p-1, s}\left(1-\hat{\alpha}_{s-p+1} B\right) y_{t-p+1}\right]+\lambda\left[\delta_{s}+\mathrm{I}_{(t \geq \tau)} \delta_{s}^{*}\right. \\
& \left.+\beta_{1 s}(1-B) y_{t-1}+\ldots+\beta_{p-1, s}(1-B) y_{t-p+1}\right]+\varepsilon_{t}
\end{aligned}
$$


which is not estimated subject to Eq. (3). Since all the variables in Eq. (8) are (periodically) stationary, non-linear least squares applied to Eq. (8) yields a $t$-ratio for the estimate of $(1-\lambda)$ which is asymptotically distributed as standard normal. Let the $t$-ratio for the test of I in Eq. (4) against PI in Eqs. (2) and (3) be denoted as $t_{\mathrm{I}}$.

There are four possible outcomes for the non-tested testing procedure. First, $t_{\mathrm{PI}}$ is significant while $t_{1}$ is not, so PI is rejected while I is not. Hence, the integrated times series model Eq. (4) is selected. Second, $t_{1}$ is significant while $t_{\mathrm{PI}}$ is not, in which case the PI model is selected over the I model. There, neither $t_{\mathrm{PI}}$ nor $t_{1}$ is significant, so both the PI and I models may be adequate representations of the data. Fourth, both $t_{\mathrm{PI}}$ and $t_{1}$ are significant, which implies that neither of the models Eqs. (2) and (3), nor Eq. (4), adequately describes the series.

An important empirical question which now arises is the determination of the value of $\tau$. It is assumed that a PI model is estimated and it is desired to check whether Eq. (4) is more appropriate. The value of $\tau$ may be determined by applying parameter constancy tests to Eqs. (2) and (3). Such a procedure necessarily involves a series of tests, which will affect the overall significance level of the tests. An alternative strategy is to rely on visual inspection, from which it appears that changes might have occurred in the period 1972-1975. In this case, it would seem sensible to use a lower nominal significance level than usual to make some allowance for the sequential nature of the testing problem.

\section{Empirical applications}

In this section the nested and non-nested testing procedures are applied to the logarithms of quarterly real GNP series for Austria and Germany. These data have been chosen since periodically integrated models can be fitted to each of these time series.

\subsection{Austria}

Consider the quarterly real GNP series for Austria over the sample period 1964.1-1989.1 (101 observations). From the quarterly $y_{t}$ series and the annual series, it can be observed that the seasonal fluctuations seem to change slowly over time. In fact, the observations in Quarter 3 seem to shift from close to those in Quarter 4 to those in Quarter 2. Furthermore, the $y_{t}$ series display a trending pattern. Similar graphs for the corresponding $(1-B) y_{t}$ time series indicate that the $(1-B) y_{t}$ series seem to evolve in a reasonably constant manner over time, except for a possible break somewhere over the period 1972-1974.

The analysis for Austria is initiated by fitting a $\operatorname{PAR}(p)$ model. Model selection criteria, namely $F_{-}$ type tests for the significance of the coefficients at lag $p+1$, indicate that an appropriate value for $p$ is 2 , and that some $\beta$ parameters can be set equal to zero. In Ref. [5] it is shown that this $F$-type test is most powerful in detecting the correct order of the model. Upon applying the Boswijk and Franses [1] test for a unit root, there is evidence of a single unit root, implying that an unrestricted PAR (2) process can be restricted by imposing the non-linear restriction in Eq. (5). The test statistic is a likelihood ratio test, which follows a standard Dickey-Fuller distribution under the null hypothesis. Hence, for the logarithm 
of real GNP series for Austria, the PIAR (2) model, except for the $\mu_{s}$ parameters, is estimated as follows (using the non-linear least squares routine in MicroTSP version 7.0):

$$
\begin{aligned}
& \left(1-\alpha_{s} B\right) y_{t}=\mu_{s}+\beta_{1 s}\left(1-\alpha_{s-1} B\right) y_{t-1}+\varepsilon_{t} \\
& \hat{\alpha}_{2}=\underset{(0.009)}{0.950} \hat{\alpha}_{3}=\underset{(0.008)}{0.972} \hat{\alpha}_{4}=\underset{(0.010)}{1.033} \hat{\alpha}_{1}=1 /\left(\hat{\alpha}_{2} \hat{\alpha}_{3} \hat{\alpha}_{4}\right)=\begin{array}{c}
1.048 \\
(0.011)
\end{array} \\
& \hat{\beta}_{11}=-0.473 \quad \hat{\beta}_{12}=-0.328 \\
& \text { (0.201) (0.172) }
\end{aligned}
$$

where the asymptotic standard errors are given in parentheses. Although the estimate of the $\beta_{12}$ parameter may seem insignificant, deletion of the corresponding variable results in an inadequate model through serial correlation of the residuals. An $F(3,90)$ test for the hypothesis that $\alpha_{s}=\alpha$ for all $s$ yields a value of 17.701 , which implies a rejection of the null hypothesis. This model, as well as all subsequent models, passes LM-type diagnostic checks for residual autocorrelation of orders 1 and 4, for periodic residual autocorrelation of order 1, for normality, and for the absence of ARCH effects of orders 1 and 4. Given the two lags in model Eq. (9), estimation uses 99 observations. The number of parameter to be estimated is 9 , arising from four $\mu_{s}$, two $\beta_{s}$ and three unrestricted $\alpha_{s}$ parameters in Eq. (9).

In estimating models like Eq. (4) for the $y_{t}$ process, it is assumed that $\tau$ can be set a priori equal to either $1972.1,1973.1$ or 1974.1 , based on visual inspection of first differences and quarterly first differences. Furthermore, the estimated residuals of models such as

$$
(1-B) y_{t}=\delta_{s}+\mathrm{I}_{(t \geq \tau)} \delta_{s}^{*}+\beta_{1 s}(1-B) y_{t-1}+\varepsilon_{t}
$$

pass the standard LM-type diagnostic checks.

\begin{tabular}{|c|c|c|c|c|c|}
\hline & \multirow{2}{*}{$\begin{array}{l}\text { Known structural } \\
\text { break } \tau\end{array}$} & \multicolumn{2}{|c|}{ Nested tests } & \multicolumn{2}{|c|}{ Non-nested tests } \\
\hline & & $F_{\mathrm{PI}}$ & $F_{1}$ & $t_{\mathrm{PI}}$ & $t_{1}$ \\
\hline \multicolumn{6}{|l|}{ Austria } \\
\hline & 1972.1 & $6.200^{* *}$ & 2.761 & $5.830^{* *}$ & -0.136 \\
\hline & 1973.1 & $3.747^{* *}$ & 2.788 & $3.194^{* *}$ & 0.689 \\
\hline & 1974.1 & $4.017^{* *}$ & 2.946 & $3.351^{* *}$ & 0.871 \\
\hline \multicolumn{6}{|l|}{ Germany } \\
\hline & 1972.1 & $4.777^{* *}$ & $8.276^{* *}$ & $3.139^{* *}$ & $3.439^{* *}$ \\
\hline & 1973.1 & $3.689^{* *}$ & $7.273^{* *}$ & $2.883^{* *}$ & $2.997^{* *}$ \\
\hline & 1974.1 & $2.897^{*}$ & $6.626^{* *}$ & $2.758^{* *}$ & $2.523^{*}$ \\
\hline
\end{tabular}

The general model Eq. (5) that nests Eqs. (9) and (10) has 13 parameters to be estimated. In Table 1 are reported the corresponding values of the $F_{\mathrm{PI}}$ and $F_{1}$ test statistics for the nested case.

Table 1

Empirical results for real GNP in Austria and Germany ${ }^{1}$

\footnotetext{
* Significant at the $5 \%$ level.

** Significant at the $1 \%$ level.

${ }^{1}$ The $F_{\mathrm{PI}}$ test has $(4,86)$ and $(4,107)$ degrees of freedom for Austria and Germany, respectively, while the corresponding $F_{1}$ test has $(3,86)$ and $(3,107)$ degrees of freedom.
} 
It is clear that the PI hypothesis is rejected by significant values of the $F_{\mathrm{PI}}$ statistics against the general model. On the other hand, the $F_{\mathrm{I}}$ test statistics are not significant at conventional significance levels, so that the hypothesis is not rejected. Furthermore, the hypothesis that the $\alpha_{s}^{*}$ are equal to zero and that the $\alpha_{s}$ are equal to one is rejected, with $F$ test values of $12.882,10.653$ and 10.899 for $\tau$ at 1972.1, 1973.1 and 1974.1, respectively. In sum, these nested testing results suggest that the I model in Eq. (4) is preferred to the PI model in Eqs. (2) and (3). The evidence against the PI hypothesis seems to be most convincing when a structural break is established at 1972.1.

The non-nested testing results summarized in Table 1 indicate a similar conclusion, with the $t_{\mathrm{PI}}$ statistics all being highly significant and the $t_{\mathrm{I}}$ statistics being insignificant. Again, the evidence against the PI null hypothesis is most convincing at $\tau=1972.1$. Therefore, the model is preferred to that of the PI process. The estimation results for Eq. (10), with $\tau$ set at 1972.1 are

$$
\begin{aligned}
& \hat{\delta}_{1}=\begin{array}{l}
-0.157 \\
(0.007)
\end{array} \quad \hat{\delta}_{1}^{*}=\begin{array}{l}
0.028 \\
(0.006)
\end{array} \quad \hat{\beta}_{11}=\begin{array}{l}
-0.507 \\
(0.197)
\end{array} \\
& \hat{\delta}_{2}=\begin{array}{l}
-0.050 \\
(0.029)
\end{array} \hat{\delta}_{2}^{*}=\begin{array}{l}
-0.029 \\
(0.006)
\end{array} \quad \hat{\beta}_{12}=\begin{array}{l}
-0.337 \\
(0.161)
\end{array} \\
& \hat{\delta}_{3}=\begin{array}{r}
-0.081 \\
(0.004)
\end{array} \quad \hat{\delta}_{3}^{*}=\begin{array}{r}
-0.019 \\
(0.005)
\end{array} \\
& \hat{\delta}_{4}=-0.026 \quad \hat{\delta}_{4}^{*}=-0.016 \\
& (0.004) \quad(0.005)
\end{aligned}
$$

These parameter estimates indicate that the structural changes occurred in all four seasons, with the $\delta_{s}^{*}$ estimates all highly significant.

Finally these estimation results can be used to investigate whether the seasonal fluctuations have decreased over time (see Ref. [2] for an alternative approach to testing for changing seasonality). When the seasonal fluctuations have been constant over time, the sum of the $\delta_{s}^{*}$ equals zero. When the sum of the estimated $\delta_{s}^{*}$ parameters is significantly less than zero, the seasonal fluctuations are seen to have decreased since the difference between the quarterly observations would have become smaller. The relevant $F$ test for this null hypothesis has a value of 0.092 , which is not significant at any conventional level.

\subsection{Germany}

The second empirical example is provided by the real GNP series in Germany, observed from 1960.1-1990.4 (124 observations). From the quarterly $y_{t}$ series, the annual series, and the corresponding first differenced time series, the seasonal patterns seem to change over time. For example, it is observed that the ranking of observations changes in the sense that the observations in Quarter 3 are highest in 1960, while those in the fourth quarter are higher in 1990. There also appears to be a trending pattern in the $y_{t}$ series. 
Along similar lines as for Austria, a PIAR (2) model like Eq. (9) is fitted to the German data, with parameter estimates (except for the $\mu_{s}$ ) given by

$$
\begin{aligned}
& \hat{\alpha}_{2}=\begin{array}{l}
0.962 \\
(0.011)
\end{array} \quad \hat{\alpha}_{3}=\begin{array}{l}
0.912 \\
(0.009)
\end{array} \quad \hat{\alpha}_{4}=\begin{array}{l}
1.113 \\
(0.012)
\end{array} \quad \hat{\alpha}_{1}=1 /\left(\hat{\alpha}_{2} \hat{\alpha}_{3} \hat{\alpha}_{4}\right)=1.025 \\
& \hat{\beta}_{11}=0.338 \quad \hat{\beta}_{12}=-0.675 \quad \hat{\beta}_{13}=-0.351 \quad \hat{\beta}_{14}=-0.428 \\
& \begin{array}{llll}
(0.195) & (0.152) & (0.154) & (0.186)
\end{array}
\end{aligned}
$$

Estimates of all four $\beta_{1 \mathrm{~s}}$ parameters are given since deletion of any corresponding variables yields significant serial correlation. This model passes all LM-type diagnostic checks. An $F(3,110)$ test for the equality of the $\alpha_{s}$ parameters yields the highly significant value of 20.653 .

Visual inspection of first differences and quarterly first differences suggests that $\tau$ may be set at 1972.1, 1973.1 or 1974.1, just as in the case of Austria, with the $F_{\mathrm{PI}}$ results reported in Table 1. It is clear that the PI hypothesis is rejected against the general model in Eq. (5) at conventional significance levels. The $F_{1}$ test statistics in the second column of Table 1 indicate that the null hypothesis in Eq. (4) can also be rejected at conventional significance levels. This implies that, although both models Eqs. (2)-(4), appear to be statistically adequate representations of the data using standard diagnostic checks, the general model Eq. (5) is preferred over the two nested competitors. This empirical result for the nested case is in marked contrast to the outcome for Austria. The estimation results for the general model Eq. (5) for $\tau$ set at 1972.1 are given by

$$
\begin{aligned}
& \hat{\alpha}_{2}=\begin{array}{l}
1.022 \\
(0.022)
\end{array} \quad \hat{\alpha}_{3}=\begin{array}{l}
0.940 \\
(0.017)
\end{array} \quad \hat{\alpha}_{4}=\begin{array}{l}
1.082 \\
(0.020)
\end{array} \quad \hat{\alpha}_{1}=1 /\left(\hat{\alpha}_{2} \hat{\alpha}_{3} \hat{\alpha}_{4}\right)=0.962 \\
& \hat{\beta}_{11}=\begin{array}{l}
0.302 \\
(0.188)
\end{array} \quad \hat{\beta}_{12}=\begin{array}{l}
-0.623 \\
(0.165)
\end{array} \quad \hat{\beta}_{13}=\begin{array}{r}
-0.222 \\
(0.167)
\end{array} \hat{\beta}_{14}=\begin{array}{l}
-0.390 \\
(0.191)
\end{array} \\
& \hat{\kappa}_{1}=\begin{array}{l}
0.273 \\
(0.151)
\end{array} \quad \hat{\kappa}_{2}=\begin{array}{l}
0.021 \\
(0.079)
\end{array} \hat{\kappa}_{3}=\begin{array}{r}
-0.422 \\
(0.093)
\end{array} \hat{\kappa}_{4}=\begin{array}{l}
-0.314 \\
(0.114)
\end{array} \\
& \hat{\kappa}_{1}^{*}=0.033 \hat{\kappa}_{2}^{*}=-0.022 \hat{\kappa}_{3}^{*}=-0.014 \hat{\kappa}_{4}^{*}=0.005 \\
& \begin{array}{llll}
(0.010) & (0.010) & (0.012) & (0.010)
\end{array}
\end{aligned}
$$

These estimates are reported for illustrative purposes only. For further use of this model, several variables may be deleted since their corresponding parameter estimates appear to be insignificant. Given that the $\kappa_{s}$ and $\kappa_{s}^{*}$ parameters are functions of mean shifts and the autoregressive parameters $\alpha_{s}$ and $\beta_{s}$, it is not immediately clear what can be concluded with respect to a decreasing seasonal variation, as in the case for Austria.

The results for the non-nested testing procedure are summarized in the final two cclumns of Table 1. All $t_{\mathrm{PI}}$ and $t_{\mathrm{I}}$ test statistics are significant and indicate the rejection of the respective null hypotheses. Within the context of non-nested testing, this suggests that neither the PI nor the I model is appropriate and that each model needs to be modified. This outcome is again in contrast to the case of Austria, in which the PI model was rejected in favour of its I counterpart. Moreover, in contrast to the rejection of both the PI and I models in favour of the general model for Germany in the nested case, rejection of PI and I using non-nested tests does not suggest whether an appropriate model exists. 


\section{Acknowledgements}

The first author thanks the Royal Netherlands Academy of Arts and Sciences for it financial support. The second author wishes to acknowledge the financial support of the Australian Research Council and the Royal Netherlands Academy of Arts and Sciences. Much of this paper was written while the second author was visiting CentER at Tilburg University and the Tinbergen Institute at Erasmus University Rotterdam.

\section{References}

[1] H.P. Boswijk and P.H. Franses, Unit roots in periodic autoregresions, Journal of Time Series Analysis 17 (1994) 221245.

[2] F. Canova, and B.E. Hansen, Are seasonal patterns constant over time? A test for seasonal stability, unpublished manuscript, Brown University.

[3] R. Davidson and J.G. MacKinnon, Several tests for model specification in the presence of alternative hypotheses, Econometrica 49 (1981) 781-793.

[4] P.H. Franses and M. McAleer, Testing nested and non-nested periodically integrated autoregressive models, Paper presented at the Australasian meeting of the Econometric Society, Armidale, Australia, July, 1994. To appear in Communications in Statistics: Theory and Methods.

[5] P.H. Franses and R. Paap, Model selection in periodic autoregressions, Oxford Bulletin of Economics and Statistics 56 (1994) 421-439.

[6] M. McAleer and M.H. Pesaran, Statistical inference in non-nested econometric models, Applied Mathematics and Computation 20 (1986) 271-311. 